Continuum Mechanics and Thermodynamics manuscript No.

(will be inserted by the editor)

\title{
A Rate-Dependent Two-Dimensional Free Energy Model for Ferroelectric Single Crystals
}

\author{
Sang-Joo Kim ${ }^{1}$, Stefan Seelecke ${ }^{2}$, Brian L. Ball ${ }^{3}$, Ralph C. Smith ${ }^{3}$ \\ 1 Dept. of Mechanical and Information Engineering, University of Seoul, Korea \\ e-mail: sjk@uos.ac.kr \\ 2 Center for Research in Scientific Computation, Dept. of Mechanical \& Aerospace Engineering, North Carolina State \\ University, USA \\ e-mail: stefan_seelecke@ncsu.edu - corresponding author \\ 3 Center for Research in Scientific Computation, Dept. of Mathematics, North Carolina State University, USA \\ e-mail: blball@ncsu.edu,rsmith@ncsu.edu
}

The date of receipt and acceptance will be inserted by the editor

\begin{abstract}
The one-dimensional free energy model for ferroelectric materials developed by Smith et al. [28-30] is generalized to two dimensions. The two-dimensional free energy potential proposed in this paper consists of four energy wells that correspond to four variants of the material. The wells are separated by four saddle points, representing the barriers for $90^{\circ}$-switching processes, and a local maximum, across which $180^{\circ}$-switching processes take place. The free energy potential is combined with evolution equations for the variant fractions based on the theory of thermally activated processes. The model is compared to recent measurements on $\mathrm{BaTiO}_{3}$ single crystals by Burcsu et al. [8], and predicitions are made concerning the response to the application of in-plane multi-axial electric fields at various frequencies and loading directions. The kinetics of the $90^{\circ}$ - and $180^{\circ}$-switching processes are discussed in detail.
\end{abstract}

Key words: ferroelectric materials -2 -D free energy model - hysteresis - rate-dependent effects

\section{Introduction}

The capability of piezoelectric materials such as barium titanate, PZT and PLZT ceramics to both actuate and sense is due to the non-centrosymmetric nature of the compounds. In actuator mode, an applied electric field changes the ionic structure of the material, resulting in reversible or irreversible strains. The application of a mechanical stress also alters the ionic configuration generating the voltages measured in piezoelectric sensors. The two mechanisms are called the converse and direct piezoelectric effects, respectively. The high sensitivity and repeatibility of the piezoelectric effects make the materials the current choice for applications such as nanopositioning, thin-film-based micro-pumping and sensing. However, the polar mechanisms which provide piezoelectric materials with the dual converse and direct effects and extreme electromechanical sensitivity, also produce varying degrees of hysteresis and constitutive nonlinearities. Both, the hysteresis and the constitutive nonlinearities must be accommodated for high performance applications utilizing piezoelectric actuators and sensors, and for this purpose an accurate and efficient model of the material behavior has to be developed.

In principle, there have been two approaches to modeling of the nonlinear behavior of piezoelectric materials, one is a macroscopic phenomenological approach and the other one a mesoscopic latticebased approach. In the former, a certain number of macroscopic internal variables are chosen to 
represent experimental measurements. An early modeling attempt in this category was made by Chen [9] and Chen and Madsen [10]. Their work uses a single scalar internal state variable that represents the extent of alignment of dipoles. A detailed account of the thermodynamic structure of phenomenological models for ferroelectricity is given by Bassiouny et al. [4,5] and Bassiouny and Maugin [6,7]. Their thermodynamic formulation uses the concept of internal variables to capture a yield function that defines the domain of reversible behavior within the space of applied fields. Recently, Cocks and McMeeking [13] have developed a phenomenological model that simulates the nonlinear response of ferroelectrics to electrical and mechanical loading. They use the average state of remnant strain and remnant polarization in the polycrystal as internal state variables. Kamlah and Tsakmakis [18] use the remnant strain and remnant polarization for the constitutive response of ferroelectrics. However, they decompose remnant strain into two parts: one is due to remnant polarization and the other is due to ferroelastic switching. Their one-dimensional model is extended to allow multi-axial loading [19]. Following the decomposition of Kamlah and Tsakmakis [18], Kim and Kwak [21] developed a onedimensional model for the nonlinear behavior of a piezoelectric wafer by adapting a rate-dependent viscoplastic mechanical model.

The second type of approach is based on a description of the switching processes at the crystal lattice scale. In a tetragonal lattice of perovskite crystals, there are six distinct types of states. Depending on applied electric and mechanical loading, each of the six states has a different energy level, and the lattices in unstable states tend to change their polarization direction into the polarization direction of more stable states. By incorporating these features into a switching model, Hwang et al. [16] were able to reproduce the main effects found in the macroscopic response of ferroelectric polycrystals, namely: dielectric hysteresis, butterfly hysteresis in strain versus electric field, and mechanical nonlinearity. Hwang et al. argued that switching occurs when the work done by local fields during a given ferroelectric switching event exceeds a critical value. They used an averaging procedure to account for the variety of crystallographic orientations and assumed uniform stress and electric fields. However, their model showed a deficiency in a comparison with measurements of uniaxial material response [22]. In order to solve the inconsistency, Hwang et al. [17], Chen and Lynch [11,12], Huber et al. [14] incorporated the local interaction between adjacent regions of material with differing polarization states, which was neglected in the original work of Hwang et al. Kim and Jiang [20] also proposed a similar constitutive and finite element model adopting a Reuss type micromechanics assumption. Their model consists of Helmholtz free energy, switching criterion and switching kinetics; the rate of switching is assumed to be proportional to the thermodynamic driving force. All of the above models are based on rate-independent or viscoplasticity-type switching evolution equations. The mass fractions of different types of states of the materials are chosen as internal variables, and their evolutions are phenomenologically described as a function of the amount of energy released during a switching process or of a thermodynamic driving force. However, it has not been shown yet that these evolution equations are capable of simulating the transient dynamics behavior and the closure of biased minor loops in the hysteresis response of the material.

To solve this problem, Smith et al. [28-30] recently regarded ferroelectric switching as a thermally activated process and developed a one-dimensional model based on kinetic switching equations. In this approach, motivated by the shape memory alloy model developed by Müller, Achenbach, and Seelecke $[1,2,23,27]$, a one-dimensional Helmholtz free energy potential consisting of two convex energy wells and a concave energy barrier (spinodal region) between the wells is proposed as a function of polarization. Depending on the applied electric field, dipoles jump from one energy well to another according to a competition between thermal activation and energy barriers. Though their model has been successful in simulating the transient dynamics and minor loop behavior of the material, it features only two energy wells, and as a result it can not model $90^{\circ}$-switching processes.

This has been improved by Sahota [26], who extended the pure polarization model to include a 1-D strain component and a corresponding $90^{\circ}$-variant. The approach allows to account for the effect of mechanical stress in one direction; however, Sahota only gives a qualitative picture based on purely convex energies without spinodal regimes, and no comparison with experimental data is made.

In the present article, we propose an extension of the model by Smith et al. to the two-dimensional case. In contrast to the full 3-D case, this still allows a graphic representation of the relevant energy 
paraelectric

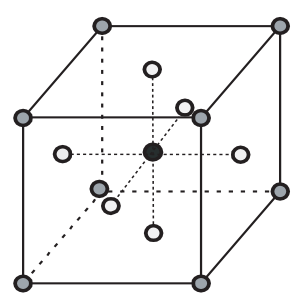

$\bullet: \mathrm{Ti}^{4+} \mathrm{O}: \mathrm{Pb}^{2+} \mathrm{O}: \mathrm{O}^{2-}$

(a)

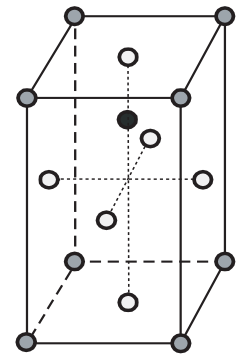

(b)

ferroelectric

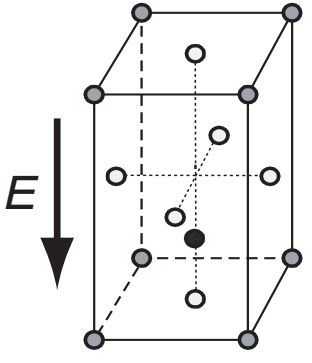

$180^{\circ}$ - switching

(c)

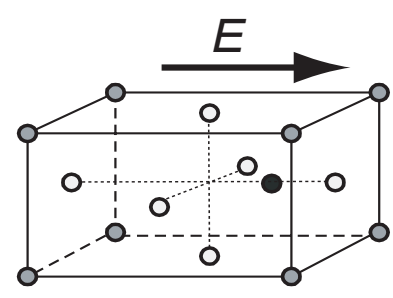

$90^{\circ}$ - switching

(d)

Fig. 1. Lattice structure in a paraelectric and ferroelectric $\mathrm{PbTiO}_{3}$ crystal, $90^{\circ}$ - and $180^{\circ}$-switching processes.

functions through surface and contour plots. In order to discuss and illustrate the complexities arising in multiple dimensions, we therefore treat the 2-D case in this paper before addressing the development of a full 3-D model at a later stage. We also confine attention to the case of pure electric loading and study the effects of mechanical stresses in a forthcoming paper [3]. We discuss the additional complexities introduced by the multi-dimensional energy landscape, e.g., the determination of transformation trajectories due to the field-dependent movement of saddle points and their impact on the transition probabilities for the transformation from one phase to the other. An extended set of evolution equations accounting for the increased number of variants is presented, which is subsequently solved numerically for several cases of electric loading. The model is compared to polarization hysteresis loops recently observed by Burcsu et al. [8] for $\mathrm{BaTiO}_{3}$ single crystals, and predictions are made for multi-axial electrical loading and various loading rates.

\section{Free Energy Function}

\subsection{Helmholtz free energy function}

Energy formulations for commonly employed ferroelectric materials can be motivated by changes occuring in the ionic structure during domain switchings or phase transitions in response to applied electric and stress fields. For illustration, we focus on $\mathrm{PbTiO}_{3}$ which is isostructural with the mineral perovskite $\left(\mathrm{CaTiO}_{3}\right)$. The crystal lattice of a perovskite material, as shown in Figure 1(a), exhibits a cubic configuration at temperatures above the Curie point $\mathrm{T}_{C}$ (paraelectric phase) and a tetragonal form below $\mathrm{T}_{C}$ (ferroelectric phase). Specifically, a unit cell of the material will have a cubic structure at temperatures $\mathrm{T}>\mathrm{T}_{C}$ with the $\mathrm{Ti}^{+4}$ ion located at the center of the lattice, and it will be in one of six tetragonal structures at temperatures $\mathrm{T}<\mathrm{T}_{C}$ with the $\mathrm{Ti}^{+4}$ ion biased along one of the three mutually orthogonal crystallographic directions, see for example Figure 1(b). Net polarization in the lattice is zero in the former case, while it has a finite value in the latter case. The position of $\mathrm{Ti}^{+4}$ is not fixed but varies under an electric field. Under an electric field opposite to the polarization direction of the lattice, the $\mathrm{Ti}^{+4}$ ion moves in the direction of the applied field and, when the electric field exceeds a critical value (coercive field), the net polarization of the lattice is reversed, Figure 1(c). This type of process is called $180^{\circ}$-switching, while the application of an electric field perpendicular to the polarization direction of the lattice, leads to what is called $90^{\circ}$-switching as shown in Figure $1(\mathrm{~d})$.

Smith et al. [30] have proposed a one-dimensional free energy potential that models $180^{\circ}$-switching processes with two energy wells and one local maximum between the two wells, as shown in Figure 2. In Figure 2, the left plot shows the one-dimensional Helmholtz free energy density $\psi_{1}\left(P_{1}\right)$, and the center and right hand side plots are the Gibbs free energy densities $g_{1}\left(P_{1} ; E_{1}\right)$ under increasing electric field. The latter are related to the Helmholtz free energy by the Legendre transformation $g_{1}=\psi_{1}-E_{1} P_{1}$. 

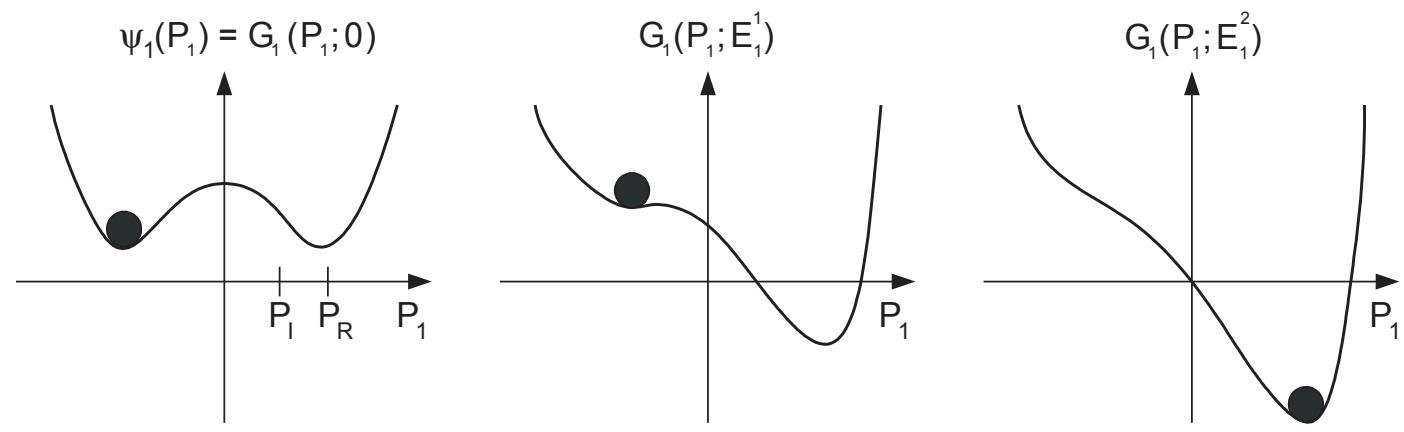

Fig. 2. One-dimensional Helmholtz free energy $\psi_{1}$ and Gibbs free energy $g_{1}$ for increasing field $E_{1}$.

The application of an electric field distorts the Gibbs energy landscape and a dipole switch occurs when the equilibrium value determining the $\mathrm{Ti}^{+4}$ position exceeds the unstable equilibrium due to the central $\mathrm{O}^{-2}$ ion pairs, as shown in the center and right plots of Figure 2.

In the present paper, the one-dimensional free energy of Smith et al. is generalized to a twodimensional free energy potential that consists of four energy wells, four saddle points and one local energy maximum. In the two-dimensional energy model, the Helmholtz free energy function per unit reference volume is given by

$$
\begin{aligned}
& \psi\left(P_{1}, P_{2}\right)=\psi_{1}\left(P_{1}\right)+\psi\left(P_{2}\right)+a P_{1}^{2} P_{2}^{2}, \\
& \psi_{1}\left(P_{1}\right)= \begin{cases}\frac{1}{2} \eta\left(P_{1}+P_{R}\right)^{2} & \text { if } P_{1} \leq-P_{I}, \\
\frac{1}{2} \eta\left(P_{I}-P_{R}\right)\left(P_{1}^{2} / P_{I}-P_{R}\right) & \text { if }-P_{I} \leq P_{1} \leq P_{I}, \\
\frac{1}{2} \eta\left(P_{1}-P_{R}\right)^{2} & \text { if } P_{I} \leq P_{1},\end{cases} \\
& \psi_{2}\left(P_{2}\right)= \begin{cases}\frac{1}{2} \eta\left(P_{2}+P_{R}\right)^{2} & \text { if } P_{2} \leq-P_{I}, \\
\frac{1}{2} \eta\left(P_{I}-P_{R}\right)\left(P_{2}^{2} / P_{I}-P_{R}\right) & \text { if }-P_{I} \leq P_{2} \leq P_{I}, \\
\frac{1}{2} \eta\left(P_{2}-P_{R}\right)^{2} & \text { if } P_{I} \leq P_{2},\end{cases}
\end{aligned}
$$

where $\psi_{1}$ and $\psi_{2}$ are the one-dimensional Helmholtz free energy potentials in the respective $P_{1^{-}}$and $P_{2^{-}}$directions that are used by Smith et al. The term $P_{I}\left(0<P_{I}<P_{R}\right)$ denotes the positive value of polarization, at which the convexity of $\psi_{1}$ and $\psi_{2}$ changes along the $P_{1^{-}}$and $P_{2^{-}}$axes, respectively, $P_{R}$ stands for the polarization value at the minima of the four energy wells, and $\eta$ is a material parameter corresponding to the inverse dielectric susceptibility of the material. The quantity $P_{R}$ is also called the remnant polarization, and $P_{I}$ is the polarization, at which the material starts to switch upon reaching the coercive electric field. The third term in (1) is a coupling term responsible for the four energy wells to be located along the $P_{1}$ and $P_{2}$ axes. Surface and contour plots for the Helmholtz free energy potential given by (1) are shown in Figures 3(a) and 3(b), respectively. In Figure 3(b), one can see four energy wells denoted by $\mathrm{W}_{1}, \mathrm{~W}_{2}, \mathrm{~W}_{3}$ and $\mathrm{W}_{4}$, each of which being located at $\left(P_{R}, 0\right),\left(0, P_{R}\right),\left(-P_{R}, 0\right)$, and $\left(0,-P_{R}\right)$ in the counterclockwise direction, respectively. In the present paper, the notations $\mathrm{W}_{1}$, $\mathrm{W}_{2}, \mathrm{~W}_{3}$ and $\mathrm{W}_{4}$ are used to denote the energy minimum states in the corresponding wells as well as the wells themselves. The four saddle points are denoted as $\mathrm{S}_{1}, \mathrm{~S}_{2}, \mathrm{~S}_{3}$ and $\mathrm{S}_{4}$ in the figure and are located at $\left(P_{C}, P_{C}\right),\left(-P_{C}, P_{C}\right),\left(-P_{C},-P_{C}\right)$, and $\left(P_{C},-P_{C}\right)$, respectively, where $P_{C}$ is defined by

$$
P_{C} \equiv \sqrt{(\eta / 2 a)\left(P_{R}-P_{I}\right) / P_{I}} .
$$

In contrast to the 1-D case, there is no longer a unique location at which the transformation from one phase to another occurs. In fact, due to thermal fluctuations, there is an infinite number of paths across the "ridges" in the energy landscape that the lattice elements can take for the transformation from one well into another. A related problem has also been treated by Puglisi and Truskinovsky $[24,25]$ in the context of a system of transforming bi-stable elements. They illustrate the various transformation trajectories of elements with different barriers and construct Peierls energy landscapes from the projections of these trajectories across saddle points. To simplify the picture in our case, we 


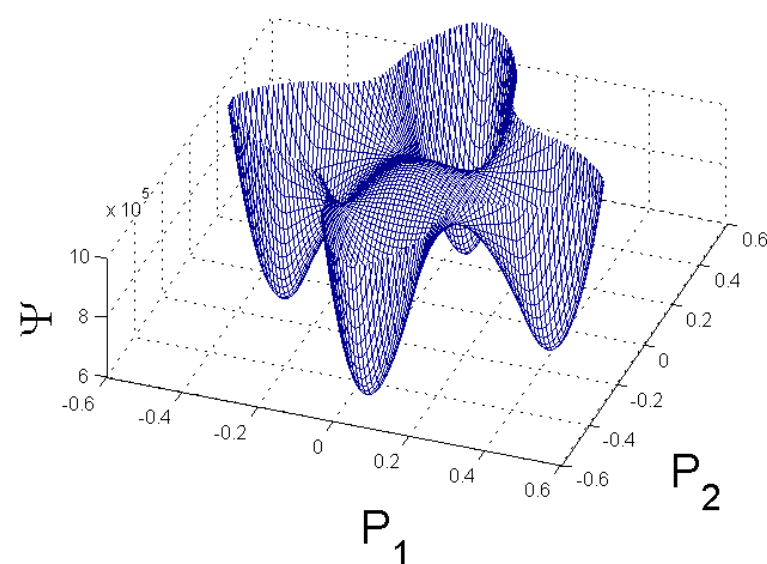

(a) Surface plot

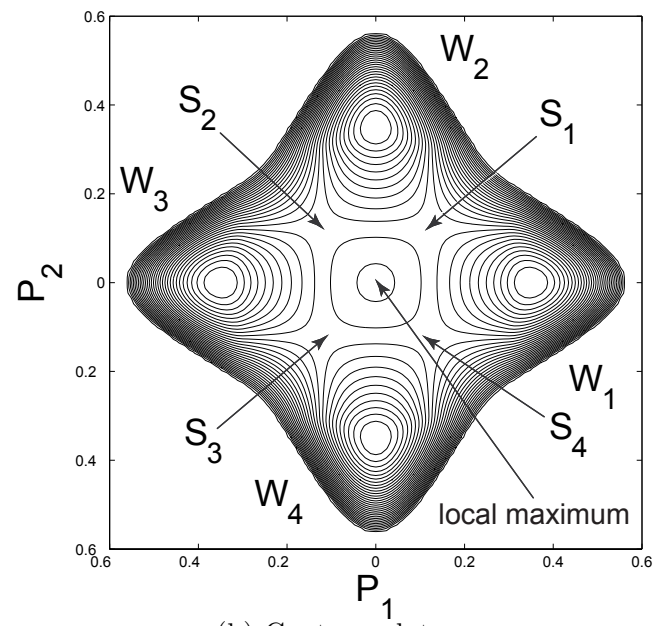

(b) Contour plot

Fig. 3. Two-dimensional Helmholtz free energy plots.

will also assume that the transformations always occur across the saddle points, as these represent the minimum energy barriers. This assumption is motivated by the observation that, even though some thermal activation is certainly present in the material, we are dealing with a solid body, and we can assume it to be relatively small compared to the barrier height of the Gibbs free energy potential. Hence, there will be only negligible fluctuation in the transformation trajectories, and the saddle points represent the energy barriers for four different types of $90^{\circ}$-domain switching processes. A local energy maximum is located at the center of the plot, and it is across this maximum that the $180^{\circ}$-domain switching processes occur. Finally, it is to be noted that the two-dimensional free energy given by (1)-(3) reduces to the one-dimensional model of Smith et al., only if the electric field is applied in the $\pm P_{1}$-direction in Figure 3 and no $90^{\circ}$-switching is allowed. In this case, only $180^{\circ}$-domain switching occurs between dipoles in $\mathrm{W}_{1}$ and $\mathrm{W}_{3}$ wells, and since $P_{2}=0$ along the $P_{1}$ axis, the two-dimensional free energy (1) reduces to $\psi\left(P_{1}, P_{2}\right)=\psi_{1}\left(P_{1}\right)+\frac{1}{2} \eta P_{R}\left(P_{I}-P_{R}\right)$.

\subsection{Gibbs free energy function}

The last section discussed the details of the Helmholtz free energy function, which, due to $E_{i}=\partial \psi / \partial P_{i}$, is the primary constitutive quantity to be determined from $\left(E_{i}, P_{i}\right)$-diagrams. The free energy that dictates the kinetics of the phase transformations, however, is the Gibbs free energy, and it is really its energy barriers and minima that are of primary interest.

In the two-dimensional model, the work of a dipole in an electric field is combined with the Helmholtz energy $\psi$ given by (1) to yield

$$
g\left(P_{1}, P_{2} ; E_{1}, E_{2}\right)=\psi\left(P_{1}, P_{2}\right)-E_{1} P_{1}-E_{2} P_{2},
$$

where $E_{1}$ and $E_{2}$ are the components of applied electric field in the $P_{1}$ and $P_{2}$ directions, respectively. If an electric field of magnitude $E$ is applied at an angle $\theta_{E}$ with respect to the positive $P_{1}$ axis in the counterclockwise direction, then $E_{1}=E \cos \theta_{E}$ and $E_{2}=E \sin \theta_{E}$. The semicolon notation used in (5) indicates that $g$ is a function of $P_{i}$ as shown in Figure 3; the electric field $E_{i}$ represents a parameter shaping the energy landscape by changing the locations of local minima, local maximum and saddle points.

\section{Basic Model Equations}

In order to describe the kinetics of the switching processes, we consider a representative volume element, composed of mesoscopic lattice elements, each of which see the energy landscape introduced above. 
Due to thermal activation, present in every physical body at non-zero temperature, the polarization of a lattice element fluctuates about its equilibrium value in the specific well, which characterizes its phase $\left( \pm 180^{\circ}, \pm 90^{\circ}\right.$ or $\left.W_{1}-W_{4}\right)$. When an electric field is applied in a certain direction, the corresponding energy barriers are lowered, and switching can occur from one state to another.

The average polarization of the representative volume element in the $i$-direction is given by

$$
\bar{P}_{i}=\sum_{\alpha=1}^{4} x_{\alpha} P_{i}^{\alpha}
$$

This expression is the weighted sum of the average polarizations of all lattice elements $P_{i}^{\alpha}$ in the individual wells, and the weighting factors are the respective phase fractions $x_{\alpha}$. The average polarizations $P_{i}^{\alpha}$ can be computed from statistical arguments, which are based on the probability to find a particular polarization state $\left(P_{1}, P_{2}\right)$

$$
\mu\left(P_{1}, P_{2}\right)=C \exp \left\{-\frac{g\left(P_{1}, P_{2} ; E_{1}, E_{2}\right)}{k_{B} T / V_{L E}}\right\} .
$$

This is a typical Boltzmann expression with a normalization constant $C, k_{B}$ and $T$ are Boltzmann's constant and the absolute temperature, $g$ is the Gibbs free energy density introduced above, and $V_{L E}$ is the volume of a lattice element. The average polarization in phase $\alpha$ is then obtained from

$$
P_{i}^{\alpha}=\iint P_{i} \mu\left(P_{1}, P_{2}\right) d P_{1} d P_{2},
$$

with the domain of integration extending according to the limits used in the energy definition (2) and (3). In the limiting case of vanishing thermal activation, the probabilities degenerate to Dirac-Delta functions, and the average values coincide with the polarizations at the minima of the energy wells.

The phase fractions $x_{\alpha}$ in Eq. (6) are determined from a set of evolution equations, motivated by the theory of thermally activated processes. The rate of change of the phase fraction in energy well $\mathrm{W}_{\alpha}$ is given by

$$
\dot{x}_{\alpha}=\sum_{\beta=1, \beta \neq \alpha}^{4}\left(\mu_{\beta \alpha} x_{\beta}-\mu_{\alpha \beta} x_{\alpha}\right) \quad \alpha=1,2,3,4,
$$

which expresses the fact that $x_{\alpha}$ can change due to a gain or loss of lattice elements from or to neighboring wells. These gains and losses are proportional to the number of lattice elements in the neighboring wells, $x_{\beta}$, and the phase fraction in the well of interest, $x_{\alpha}$. The proportionality factors $\mu_{\alpha \beta}$ are the transition probabilities for a switch from $\mathrm{W}_{\alpha}$-well to $\mathrm{W}_{\beta}$-well, and they are computed from statistical thermodynamics as

$$
\mu_{\alpha \beta}=\frac{1}{\tau_{\alpha \beta}} \exp \left(\frac{-\Delta g_{\alpha \beta}}{k_{B} T / V_{L E}}\right), \quad \alpha, \beta=1,2,3,4, \quad \alpha \neq \beta .
$$

They are the product of the probability of finding a lattice element on top of the barrier between $\mathrm{W}_{\alpha^{-}}$ and $\mathrm{W}_{\beta}$-wells, and the frequency $1 / \tau_{\alpha \beta}$ with which it attempts to cross this barrier. It is well known from statistical mechanics that these frequencies depend on the curvature of the energy well, and if this well is not rotationally symmetric, the frequencies will be anisotropic. For the sake of simplicity, we will not update the frequencies, when the positions of the saddle points move, and we will assume $\tau_{90}=\tau_{180} \equiv \tau$ for two different relaxation times $\tau_{90}$ and $\tau_{180}$, representing different time scales for the two types of switching processes. The term $\Delta g_{\alpha \beta}:=g\left(P_{i}^{\alpha \beta} ; E_{j}\right)-g\left(P_{i}^{\alpha} ; E_{j}\right)$ is the difference between the energy on top of the barrier at $P_{i}^{\alpha \beta}$ and the energy at the minimum of $\mathrm{W}_{\alpha}$-well, $P_{i}^{\alpha}$. Note that expression (10) is again the low thermal activation limit of the transition probability, based on the same assumption that was already introduced with the definition of the saddle point as the actual barrier.

The saddle points, local maximum and local minima are not fixed but move in the $\left(P_{1}, P_{2}\right)$-plane depending on the applied electric field. Their locations can be found by solving the coupled nonlinear algebraic equations, $\partial g / \partial P_{1}=0$ and $\partial g / \partial P_{2}=0$, because the gradient of the Gibbs free energy has to be zero in the $P_{1^{-}}$and $P_{2}$-directions at the extremum points. Usually, multiple solutions exist in the simultaneous equations, and we determine the type of extremum point from the following criteria: 


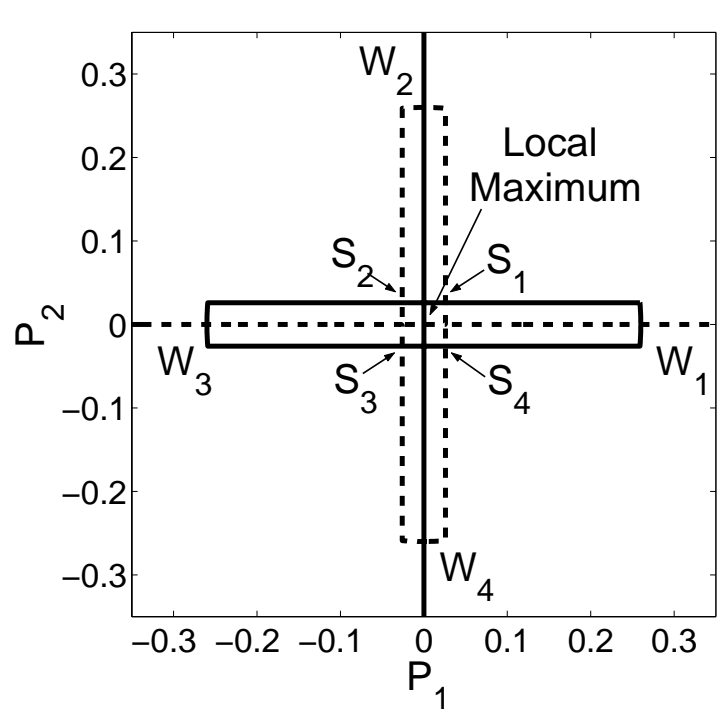

(a) $E=0$

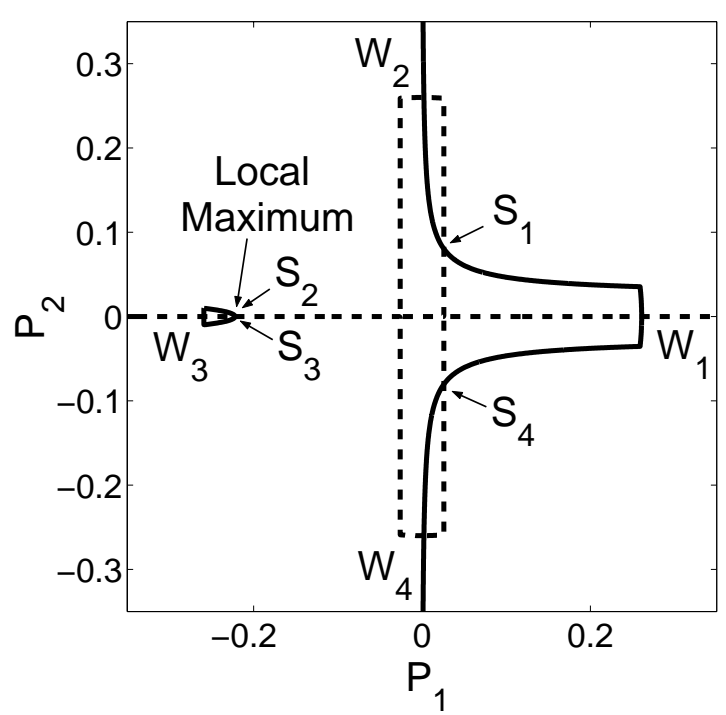

(c) $E_{2}>E_{1}$

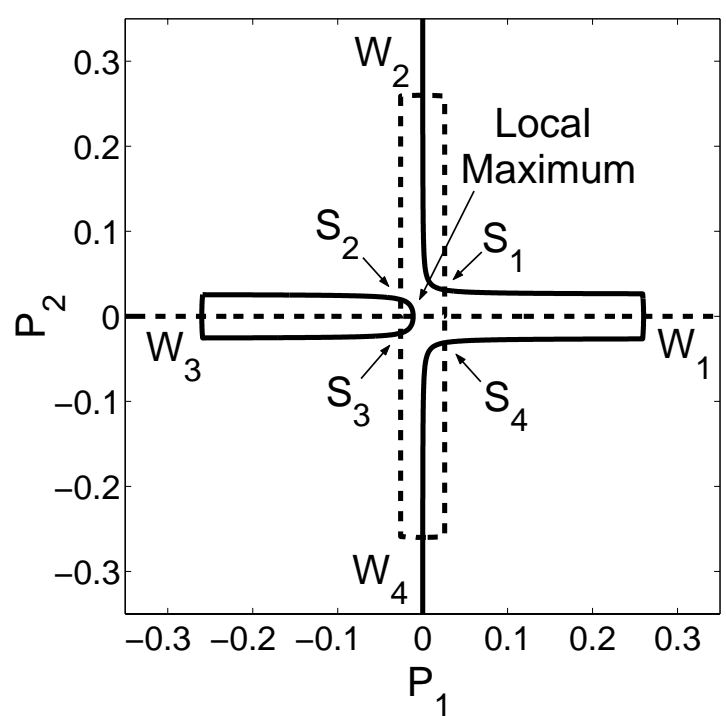

(b) $E_{1}>0$

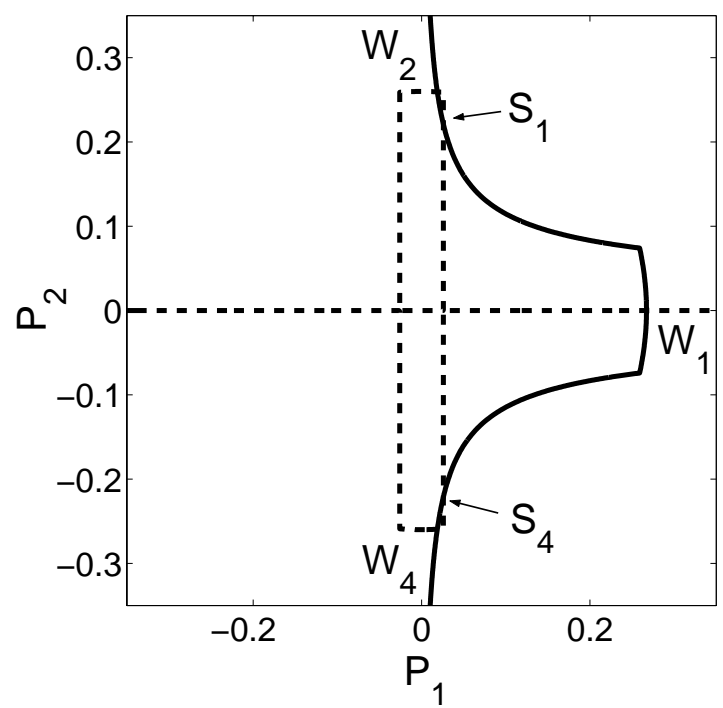

(d) $E_{3}>E_{2}$

Fig. 4. Location of extremum points of Gibbs free energy under increasing electric fields applied in the $+P_{1}$-direction.

$$
\begin{array}{lll}
\text { If } D\left(P_{1}, P_{2}\right)>0 \text { and } A\left(P_{1}, P_{2}\right)>0 & \Longrightarrow & \text { local minimum at }\left(P_{1}, P_{2}\right), \\
\text { If } D\left(P_{1}, P_{2}\right)>0 \text { and } A\left(P_{1}, P_{2}\right)<0 & \Longrightarrow & \text { local maximum at }\left(P_{1}, P_{2}\right), \\
\text { If } D\left(P_{1}, P_{2}\right)<0, & \Longrightarrow & \text { saddle point at }\left(P_{1}, P_{2}\right),
\end{array}
$$

where $D$ and $A$ are discriminant equations defined by

$$
D \equiv\left|\begin{array}{cc}
\frac{\partial^{2} G}{\partial P_{1}^{2}} & \frac{\partial^{2} G}{\partial P_{1} \partial P_{2}} \\
\frac{\partial^{2} G}{\partial P_{1} \partial P_{2}} & \frac{\partial^{2} G}{\partial P_{2}^{2}}
\end{array}\right|, \quad A \equiv \frac{\partial^{2} G}{\partial P_{1}^{2}} .
$$

The evaluation of these equations shows that the characteristics of the energy landscape may change drastically during the course of a loading/unloading process. Hence, we have to carefully analyze the landscape in order to define the correct energy barriers and the corresponding transition probabilities. The sequence displayed in Figure 4 illustrates several degeneracies that may occur, and their treatment is discussed in the sequel. 
The figure shows the movement of various extremal points while the electric field increases in the $+P_{1}$-direction. In the figure, the solid line represents $\partial G / \partial P_{1}=0$ and the dashed line $\partial G / \partial P_{2}=0$. Therefore, the points at which the solid and dashed lines intersect are extremal points. Figure 4(a) shows the various extremal points at zero electric field. At $E=E_{1}$, the saddle points $\mathrm{S}_{2}$ and $\mathrm{S}_{3}$ move vertically toward the $P_{1}$-axis and the local maximum moves leftward. They meet at $\left(-P_{C}, 0\right)$, and the local maximum converts to a saddle point. After that, the common saddle point moves leftward on the $P_{1}$-axis until it meets the local minimum $\mathrm{W}_{3}$ at $\left(-P_{I}, 0\right)$, as shown in Figure $4(\mathrm{c})$. During this period of time, the energy barriers for the $90^{\circ}$-switching processes between the $\mathrm{W}_{2^{-}}$( or $\mathrm{W}_{4^{-}}$) well and the $\mathrm{W}_{3}$-well as well as for all $180^{\circ}$-switching processes are evaluated at the common saddle point. If the electric field is further increased, the local minimum $\mathrm{W}_{3}$ and the common saddle point merge at the border between the definition ranges of the piecewise energy function and subsequently vanish. In this case, the values of minimum Gibbs energy and energy barriers are evaluated at the same location $\left(-P_{I}, 0\right)$. As a result, the transition probabilities calculated by $(10)$ for the switching processes from the $\mathrm{W}_{3}$-well to the $\mathrm{W}_{1^{-}}, \mathrm{W}_{2^{-}}$or $\mathrm{W}_{4}$-wells have maximum values, i.e., $\mu_{31}=\mu_{32}=\mu_{34}=1 / \tau$. On the other hand, the saddle point $\mathrm{S}_{1}$ moves up to $\left(P_{C},+P_{I}\right)$ and merges with the local minimum $\mathrm{W}_{2}$ when the field is increased, as shown in Figure 4(d). After this, the local minimum $\mathrm{W}_{2}$ and the saddle point $\mathrm{S}_{1}$ do no longer exist, and the transition probability for the $90^{\circ}$-switching from the $\mathrm{W}_{2}$-well to the $\mathrm{W}_{1}$-well is $\mu_{21}=1 / \tau$, because the values of minimum energy in the $\mathrm{W}_{2}$-well and the energy barrier for $90^{\circ}$-switching between $\mathrm{W}_{2}$-well and $\mathrm{W}_{1}$-well are evaluated at the same location $\left(P_{C},+P_{I}\right)$. The same argument holds for switching between the $\mathrm{W}_{4}$-well and the $\mathrm{W}_{1}$-well. Comparing Figures $4(\mathrm{c})$ and $4(\mathrm{~d})$, we see that the transition probabilities $\mu_{32}$ and $\mu_{34}$ reach their maximum value $1 / \tau$ at about $E=E_{2}$, but those of $\mu_{21}$ and $\mu_{41}$ have the same maximum value at about $E=E_{3}$. This means that $90^{\circ}$-switching in the later period of a process proceeds at a much slower speed than the $90^{\circ}$-switching in the early period.

It is evident that the determination of the transition probabilities in the multi-dimensional case requires careful tracking of the loading process. Once determined, the $\mu_{\alpha \beta}$ are then used to assemble

the RHS of the ODE system (9). Using the phase fraction constraint $\sum_{\alpha=1}^{4} x_{\alpha}=1$, we can finally reduce the four differential equations (9) to a set of three coupled differential equations:

$$
\begin{aligned}
& \dot{x}_{1}=-\left(\mu_{12}+\mu_{13}+\mu_{14}+\mu_{41}\right) x_{1}+\left(\mu_{21}-\mu_{41}\right) x_{2}+\left(\mu_{31}-\mu_{41}\right) x_{3}+\mu_{41}, \\
& \dot{x}_{2}=\left(\mu_{12}-\mu_{42}\right) x_{1}-\left(\mu_{21}+\mu_{23}+\mu_{24}+\mu_{42}\right) x_{2}+\left(\mu_{32}-\mu_{42}\right) x_{3}+\mu_{42}, \\
& \dot{x}_{3}=\left(\mu_{13}-\mu_{43}\right) x_{1}+\left(\mu_{23}-\mu_{43}\right) x_{2}-\left(\mu_{31}+\mu_{32}+\mu_{34}+\mu_{43}\right) x_{3}+\mu_{43} .
\end{aligned}
$$

Complemented by suitable initial conditions, this ODE system can be integrated numerically for a prescribed electric field input to yield the phase fraction evolution, and together with the algebraic equation (6), the time-dependent polarization can be computed. Note that the character of the ODEs introduces a natural time scale through the common relaxation time $\tau$, which allows to predict ratedependent effects in a natural way.

\section{Results and Discussions}

In this section, the response of the model described in the previous sections is calculated for several different cases of pure electric loading. We consider a single crystalline material with crystallographic direction coinciding with the global $\left(P_{1}, P_{2}\right)$ coordinate frame. Therefore, four kinds of dipoles exist in the material with their crystallographic $c$ axes in the $\pm P_{1^{-}}$and $\pm P_{2}$-axes, respectively. First, we simulate a quasistatic polarization hysteresis loop of a $\mathrm{BaTiO}_{3}$ single crystal recently observed by Burcsu et al. [8], see Figure 5. The material parameters in (14) are chosen to fit the experimental observation.

$$
\begin{gathered}
\tau=1.3 \times 10^{-2} \mathrm{sec}, \quad P_{R}=0.26 \mathrm{C} / \mathrm{m}^{2}, \quad \eta=7 \times 10^{7} \mathrm{~J} \cdot \mathrm{m} / \mathrm{C}^{2}, \\
V=1.5 \times 10^{-24} \mathrm{~m}^{3}, P_{I}=0.259 \mathrm{C} / \mathrm{m}^{2}, a=2 \times 10^{8} \mathrm{~J} \cdot \mathrm{m}^{5} / \mathrm{C}^{4}, \\
T=298^{0} \mathrm{~K} .
\end{gathered}
$$




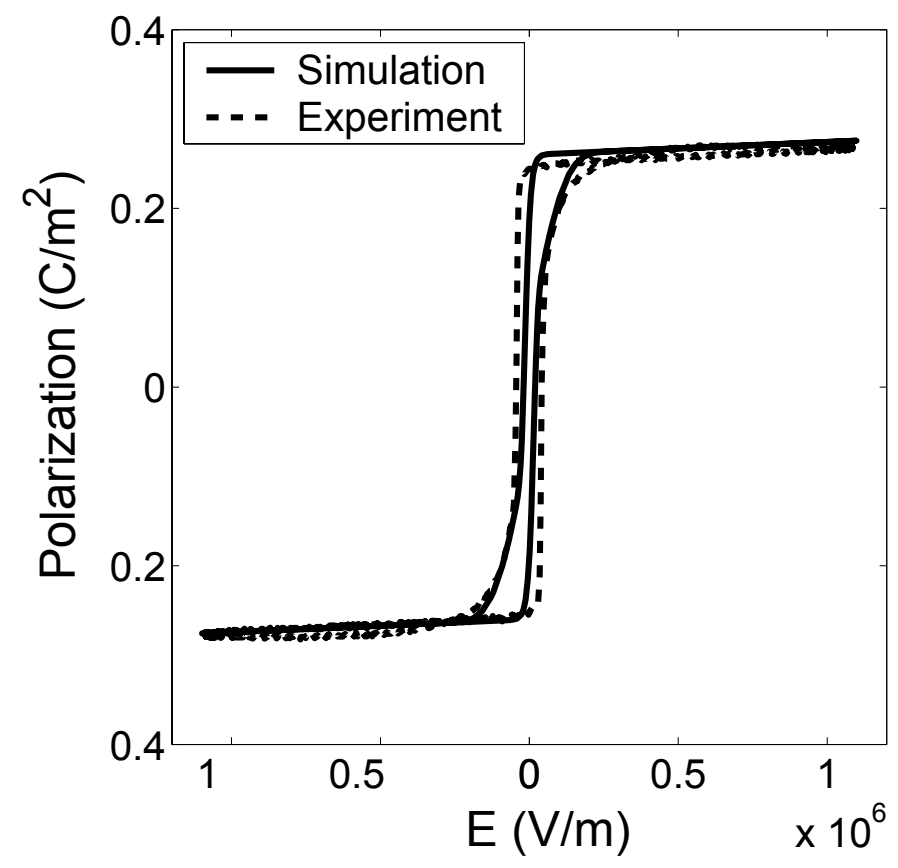

Fig. 5. Quasistatic polarization hysteresis of a $\mathrm{BaTiO}_{3}$ single crystal, measurements by Burcsu et al. $[8],\left(\theta_{E}=0, f_{E}=\right.$ $0.05 \mathrm{~Hz})$.

The applied electric field is sinusoidal, and its amplitude and frequency are $E_{a m p}=1.1 \mathrm{MV} / \mathrm{m}$ and $f_{E}=0.05 \mathrm{~Hz}$, respectively. The angle of the applied electric field is denoted by $\theta_{E}$, where $\theta_{E}$ is measured from the $+P_{1}$ axis in the counterclockwise direction. In Figure 5, the solid line represents the result of the simulation and the dashed line the measured hysteresis loop. The hysteresis loop itself exhibits a very sharp transition, typical for a single crystal, and it is rather thin, indicative of a low coercive field. Toward the end of the transformation, the slope decreases smoothly until saturation is reached. We will show this feature to be a hint at the potential micro-mechanism of the transformation, which presents itself as a two-step process with different kinetics due to the presence of $90^{\circ}$ - and $180^{\circ}$-switching.

We will first show that the 1-D two-variant model by Smith et al. is not able to capture this behavior. The prediction of the one-dimensional model can be obtained from the two-dimensional model by restraining all $90^{\circ}$-switching processes, that is, by allowing $180^{\circ}$-switching only. Identical material parameter values are used for the one-dimensional calculation, and in Figure 6, we compare the response of the two-dimensional model (solid line) with that of the one-dimensional model (dashed line). While the 2-D model reproduces the measured change in slope toward the end of the transformation, the 1-D version predicts a constant value for the coercive field. This can be understood by comparing the evolution of the phase fractions in both cases. Figure 7(a) shows these evolutions over an electric field range from $-0.1 \mathrm{MV} / \mathrm{m}$ to $0.7 \mathrm{MV} / \mathrm{m}$ in the one-dimensional case, and Figure 7(b) shows the two-dimensional case. Comparing the two figures, we see that the spill-out process of lattice elements, initiated once the barrier shielding well $\mathrm{W}_{3}$ is eliminated, is identical in both cases, and they follow the same curve in the $(P, E)$-diagram initially. In the 2-D case, however, these elements do not all jump into the $\mathrm{W}_{1}$-well but into the two lateral wells $\mathrm{W}_{2}$ and $\mathrm{W}_{4}$ as well. From here, they transform further on to $\mathrm{W}_{1}$, but in a delayed manner, see again Figure $7(\mathrm{~b})$. This is due to the circumstance that they are trapped in wells, of which the barrier to $\mathrm{W}_{1}$ first has to be eliminated by further increase in field. In fact, inspection of the phase fraction diagram shows that the combined percentage of $\mathrm{W}_{2}$ and $\mathrm{W}_{4}$ fractions is as high as $70 \%$ at the maximum, and we conclude that the more realistic 2-D model predicts a two-step transformation through repeated $90^{\circ}$-switching processes, which is also well in agreement with the observation in Figure 5. 


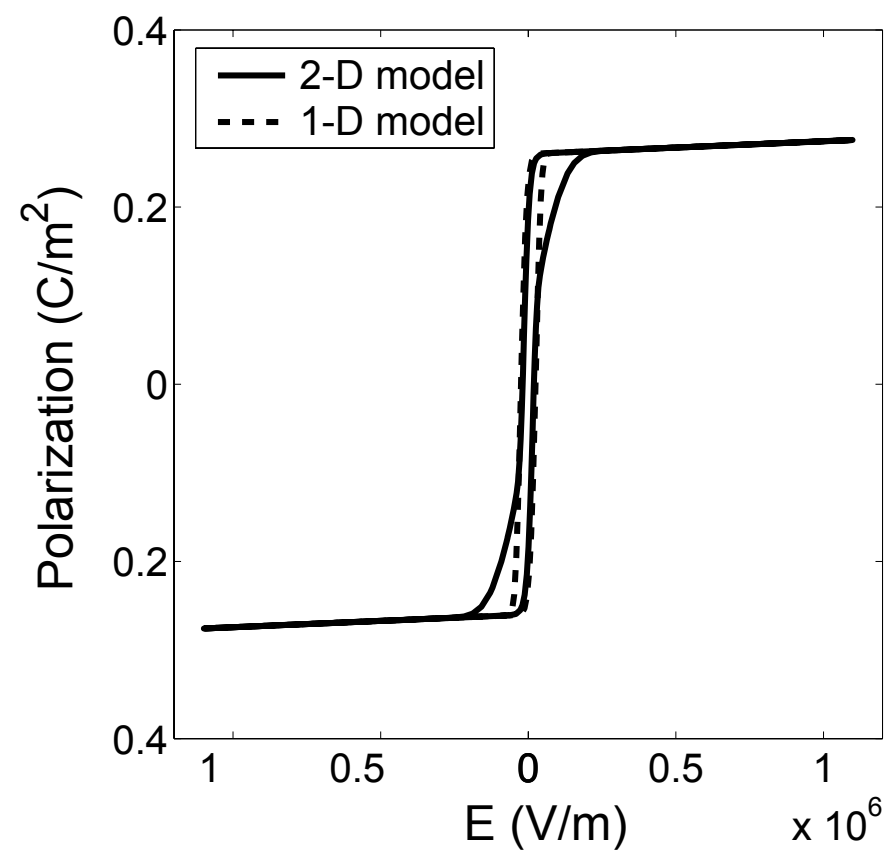

Fig. 6. Comparison of one- and two-dimensional hysteresis curves $\left(\theta_{E}=0, f_{E}=0.05 \mathrm{~Hz}\right)$.

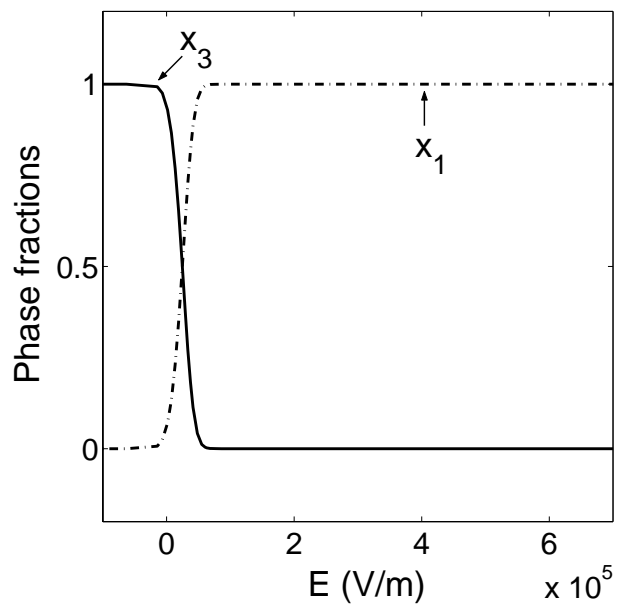

(a) One-dimensional model

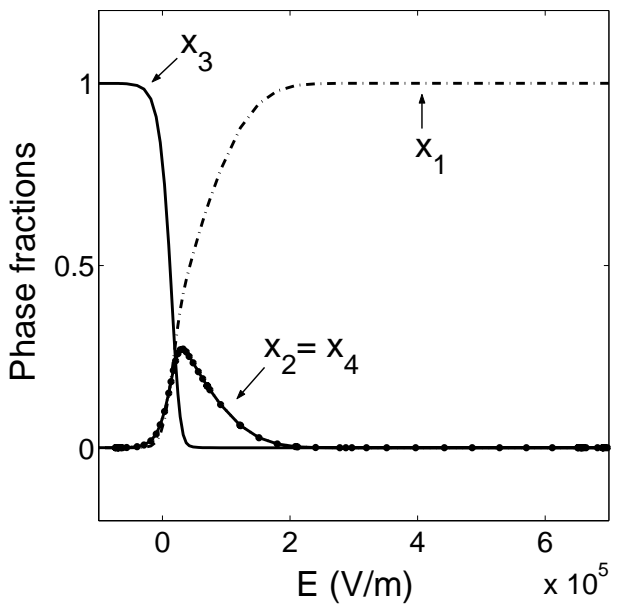

(b) Two-dimensional model

Fig. 7. Evolution of phase fractions in (a) one- and (b) two-dimensional model $\left(\theta_{E}=0, f_{E}=0.05 \mathrm{~Hz}\right)$

Next, we discuss the response of the model to electric fields applied in three different directions $\theta_{E}=0, \pi / 8$ and $\pi / 4$. We set the initial phase fractions to $\left.\left\{x_{1}, x_{2}, x_{3}, x_{4}\right\}\right|_{t=0}=\{0,0,1,0\}$, and therefore, initially, all lattices in the material are in the $\mathrm{W}_{3}$-well. The predicted polarization hystereses during the first loading cycle, with polarizations calculated in the direction of applied fields, are shown in Figure 8, and the corresponding plots of phase fraction evolution in Figure 9. In Figure 8, the solid line corresponds to the electric field of $\theta_{E}=0$, the dashed one to $\theta_{E}=\pi / 8$, and the dashed and dotted one to $\theta_{E}=\pi / 4$. The memory of the initial state is quickly erased, and we would observe steady-state hysteresis loops after the first cycle in all three cases. It is shown in Figure 8 that the value of maximum polarization is the largest for the electrical load at $\theta_{E}=0$ and the lowest for the electrical load at $\theta_{E}=\pi / 4$. This is due to the circumstance that the maximum phase fraction values depend on the angle of applied electric field as shown in Figures 9(a) and 9(c). At the largest positive electric field, they are approximately $\{1,0,0,0\}$ and $\{0.5,0.5,0,0\}$ for $\theta_{E}=0$ and $\pi / 4$, respectively. This reflects 


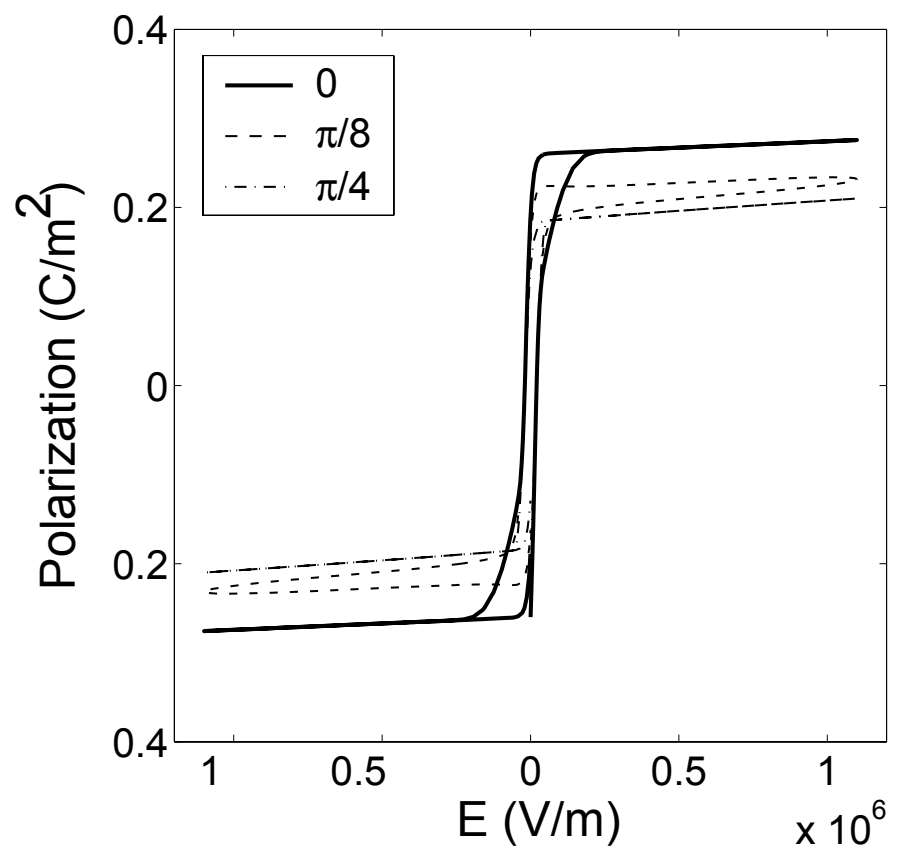

Fig. 8. Hysteresis curves at three different loading angles $\left(f_{E}=0.05 \mathrm{~Hz}\right)$.

the fact that the electric field, when applied under increasing angle from 0 to $\pi / 4$, lowers the depth of the $\mathrm{W}_{2}$-well, as much as that of the $\mathrm{W}_{1}$-well at $\theta_{E}=\pi / 4$, resulting in the equal probabilities of switching to the $\mathrm{W}_{1}$ - and $\mathrm{W}_{2}$-wells. These results are not compared to measurements by Burcsu et al., who did not study the effect of different loading direction, and probably are most closely related to the observations by Huber and Fleck [15], who studied loading in fixed directions, but with specimens cut under different orientations.

Finally, in Figure 10, we show the effect of loading rate on the polarization hysteresis. In this case, too, there are no measurements available for comparison yet, and the results have more of a predictive character. We apply the electric loading at three different frequencies, i.e., at $f_{E}=0.05 \mathrm{~Hz}, 0.3 \mathrm{~Hz}$ and $1 \mathrm{~Hz}$, and Figures. 11(a), (b) and (c) show the evolution of phase fractions at the respective loading frequencies vs. electric field in the range from $E=-0.1 \mathrm{MV} / \mathrm{m}$ to $E=0.7 \mathrm{MV} / \mathrm{m}$. In Figure 10, it is seen that the hysteresis loop gets wider with increasing loading frequency. This effect is small in the initial phase of the transformations, but it becomes very pronounced during the later period of the switching processes, when $90^{\circ}$-switchings occur from the $\mathrm{W}_{2^{-}}$and $\mathrm{W}_{4}$-wells to the $\mathrm{W}_{1^{-}}$- or $\mathrm{W}_{3}$-wells. Figure 11 shows that the rate of $90^{\circ}$-switching from $\mathrm{W}_{2^{-}}$and $\mathrm{W}_{4}$-wells to the $\mathrm{W}_{3}$-well gets slower, which reflects the inertia of the transformation. This behavior is dictated by the ratio of the relaxation times $\tau_{180 / 90}$ and the rate of loading and has to be determined from experimental data.

\section{Conclusions}

The one-dimensional free energy model for ferroelectrics by Smith et al. is generalized to a twodimensional model that consists of four energy wells, four saddle points and one energy maximum. In the proposed model, polarized mesoscopic lattice elements jump from one energy well to another across barriers between neighboring wells. Evolution equations for the four relevant variant phase fractions are derived based on ideas from the theory of thermally activated processes and statistical thermodynamics. The corresponding transition probabilities are the product of a Boltzmann term giving the probability to reach the top of an energy barrier and an attempt frequency, which is the inverse of a characteristic relaxation time. The effect of an applied electric field on the multi-dimensional energy landscape and the barriers in particular is discussed in detail, along with the algorithm for the determination of the transition probabilities. 

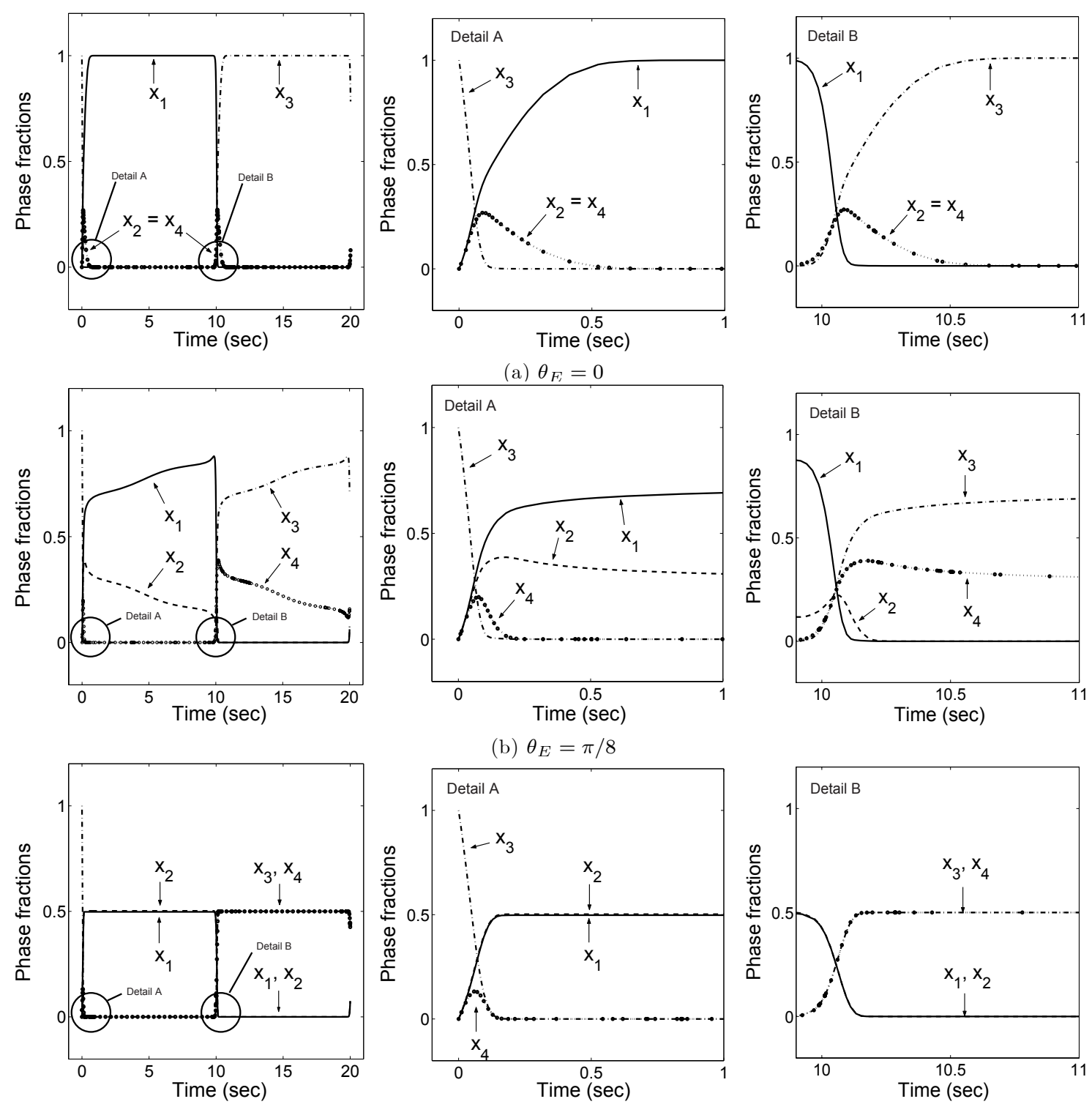

(c) $\theta_{E}=\pi / 4$

Fig. 9. Evolution of phase fractions at three different loading angles $\left(f_{E}=0.05 \mathrm{~Hz}\right)$

The prediction of the model is compared with a recently observed polarization hysteresis curve for a $\mathrm{BaTiO}_{3}$ single crystal. Using the material parameters determined from the comparison, the response of the model to multiaxial in-plane electric field loading at various frequencies is calculated. The simulations show that the response of the model strongly depends on the direction of the applied electric field. The largest polarization is obtained when the electric field is applied at $\theta_{E}=0$, i.e., in one of the principal directions of the free energy potential, and the smallest at $\theta_{E}=\pi / 4$. The model also predicts effects of loading rate, and it is observed that the hysteresis loops become wider with increasing loading frequency, this effect being particularly remarkable during the later stage of the switching process. Inspection of the variant fraction evolution reveals that switching takes place as a two-stage process with the kinetics being determined by the energy landscape and the relaxation times.

In another paper [3], the implementation of mechanical stresses and the extension to polycrystalline behavior is discussed, and a three-dimensional version of the model is in development. 


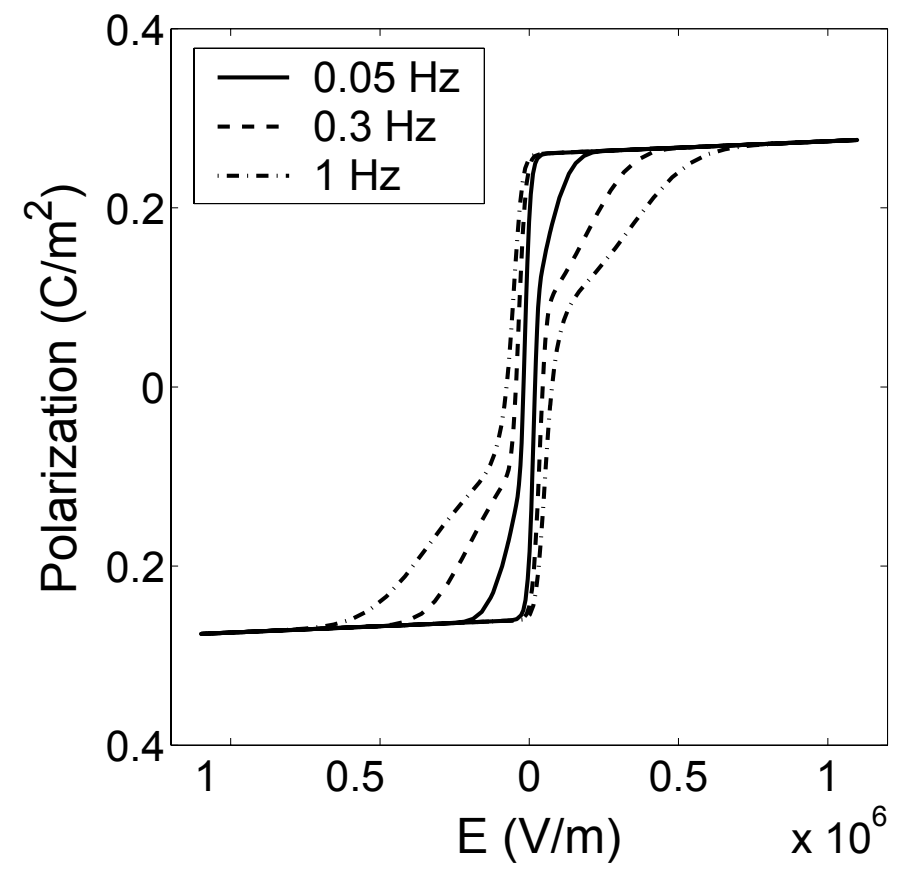

Fig. 10. Hysteresis curves at three different loading rates $\left(\theta_{E}=0\right)$.

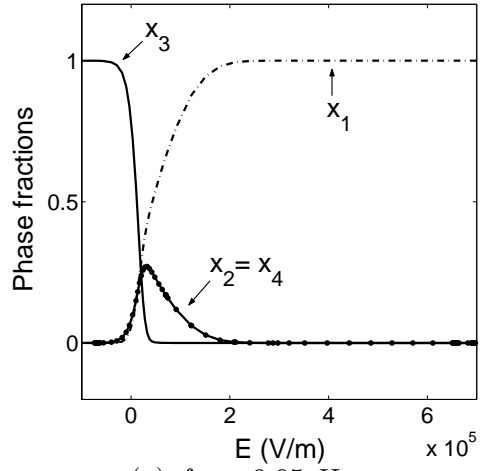

(a) $f_{E}=0.05 \mathrm{~Hz}$

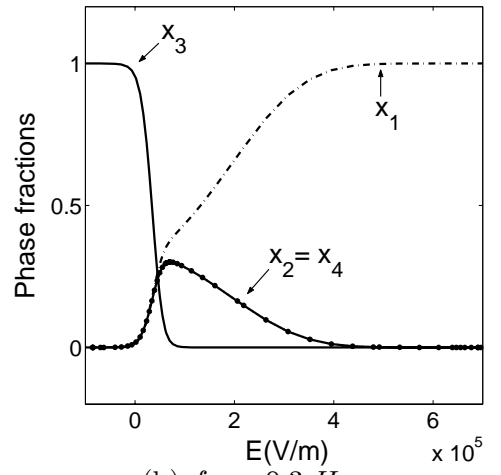

(b) $f_{E}=0.3 \mathrm{~Hz}$

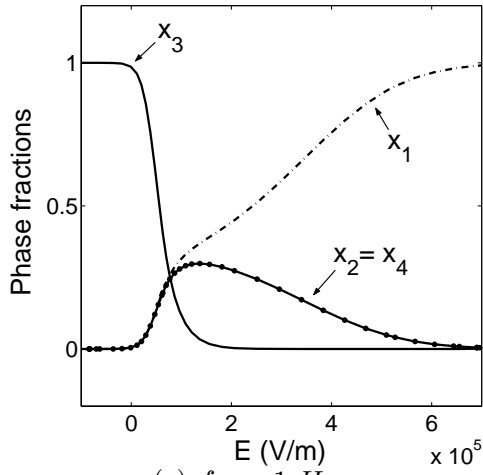

(c) $f_{E}=1 \mathrm{~Hz}$

Fig. 11. Evolution of phase fractions at three different loading rates $\left(\theta_{E}=0\right)$

\section{Acknowledgements}

The work of SS and SJK has been partially supported by the National Science Foundation through grant DMII-0134464. The research of BLB and RCS was supported through the NSF grant CMS0099764 and the Air Force Office of Scientific Research under the grants AFOSR-F49620-01-1-0107 and AFOSR-FA9550-04-1-0203.

\section{References}

1. M. Achenbach and I. Müller, "Simulation of material behavior of alloys with shape memory", Arch. Mech., 37 (6), $1985,573-585$

2. M. Achenbach, "A model for an alloy with shape memory", Int. J. Plast., 5, 1989, 371-395

3. B. L. Ball, R. C. Smith, S. J. Kim and S. Seelecke, "A Stress-Dependent Hysteresis Model for Ferroelectric Materials", J. Intell. Mat. Sys. Struct., 2005, submitted

4. E. Bassiouny, A. F. Ghaleb and G. A. Maugin, 1988a, "Thermodynamic relations for coupled electromechanical hysteresis effects-I. Basic equations", Int. J. Engng. Sci. 26, 1279-1295. 
5. E. Bassiouny, A. F. Ghaleb and G. A. Maugin, 1988b, "Thermodynamic relations for coupled electromechanical hysteresis effects-II. Poing of ceramics", Int. J. Engng. Sci. 26, 1297-1306.

6. E. Bassiouny and G. A. Maugin, 1989a, "Thermodynamical formulation for coupled electromechanical hysteresis effects-III. Parameter identification", Int. J. Engng. Sci. 27, 975-987.

7. E. Bassiouny and G. A. Maugin, 1989b, "Thermodynamical formulation for coupled electromechanical hysteresis effects-IV. Combined electromechanical loading", Int. J. Engng. Sci. 27, 989-1000.

8. E. Burcsu, G. Ravichandran and K. Bhattacharya, 2004, "Large electrostrictive actuation of barium titanate single crystals", J. Mech. Phys. 52, 823-846.

9. P. J. Chen, 1980, "Three dimensional dynamic electromechanical constitutive relations for ferroelectric materials", Int. J. Solids Struct. 16, 1059-1067.

10. P. J. Chen and M. M. Madsen, 1981, "One dimensional polar responses of the electrooptic ceramic PLZT $7 / 65 / 35$ due to domain switching", Acta Mech. 41, 255-264.

11. W. Chen and C. S. Lynch, 1998a, "A micro-electro-mechanical model for polarization switching of ferroelectric materials", Acta Mater. 46, 5303-5311.

12. W. Chen and C. S. Lynch, 1998b, "A model for simulating polarization switching and AF-F phase changes in ferroelectric ceramics", J. Intell. Mater. Systems Struct. 9, 427-431.

13. A. C. F. Cocks and R. M. McMeeking, 1999, "A phenomenological constitutive laws for the behavior of ferroelectric ceramics", Ferroelectrics 228, 219-228.

14. J. E. Huber, N. A. Fleck, C. M. Landis and R. M. McMeeking, 1999, "A constitutive model for ferroelectric polycrystals", J. Mech. Phys. Solids 47, 1663-1697.

15. J. E. Huber and N. A. Fleck, 2001, "Multi-axial electrical switching of a ferroelectric: theory versus experiment", $J$. Mech. Phys. Solids 49, 785-811.

16. S. C. Hwang, C. S. Lynch and R. M. McMeeking, 1995, "Ferroelectric/ferroelastic interactions and a polarization switching model", Acta Metall. Mater. 43, 2073-2084.

17. S. C. Hwang, J. E. Huber, R. M. McMeeking and N. A. Fleck, 1998, "The simulation of switching in polycrystalline ferroelectric ceramics", J. Appl. Phys. 84, 1530-1540.

18. M. Kamlah and C. Tsakmakis, 1999, "Phenomenological modeling of the nonlinear electromechanical coupling in ferroelectrics", Int. J. Solids Struct. 36, 669-695.

19. M. Kamlah and U. Bohle, 2001, "Finite element analysis of piezoceramic components taking into account ferroelectric hysteresis behavior", Int. J. Solids Struct. 38, 605-633.

20. S. J. Kim and Q. Jiang, 2002, "A finite element model for rate-dependent behavior of ferroelectric ceramics", Int. J. Solids Struct. 39, 1015-1030.

21. S. J. Kim and M. K. Kwak, 2004, "The nonlinear behavior of a piezoelectric wafer: constitutive equations and simulation", Smart Mater. Struct. 13, 184-190.

22. C. S. Lynch, 1996, "The effect of uniaxial stress on the electro-mechanical response of 8/65/35 PLZT", Acta Mater. $44,4137-4148$.

23. I. Müller, and S. Seelecke, "Thermodynamic Aspects of Shape Memory Alloys", in Topics in the mathematical modelling of smart materials, Morro, A. (ed.), Math. Comp. Model., 34, 2001, 1307-1355

24. G. Puglisi and L. Truskinovsky, "Rate Independent Hysteresis in a Bi-stable Chain", J. Mech. Phys. Solids, 50, 165-187, 2002

25. G. Puglisi and L. Truskinovsky, "A Mechanism of Transformational Plasticity", Cont. Mech. Thermodyn., 14 (5), $437-457,2002$

26. H. Sahota, "Simulation of Butterfly Loops in Ferroelectric materials", Cont. Mech. Thermodyn., 16 (1-2), 163-176, 2004

27. S. Seelecke, and I. Müller, Shape Memory Alloy Actuators: Modeling and Simulation, ASME Appl. Mech. Rev., vol. 57, No. 1, 2004

28. R. C. Smith, S. Seelecke, Z. Ounaies, "A Free Energy Model for Piezoceramic Materials, Proceedings 9th SPIE Conference on Smart Structures and Materials, San Diego, USA, 17-22 March 2002, vol. 4693, 2002, 183-190

29. R. C. Smith, S. Seelecke, Z. Ounaies and J. Smith. "A Free Energy Model for Hysteresis in Ferroelectric Materials", J. Intell. Mat. Sys. Struct., vol 14, 11, 2003, 719-739

30. R. C. Smith, S. Seelecke, M. J. Dapino and Z. Ounaies, "A Unified Model for Hysteresis in Ferroic Materials", J. Mech. Phys. Solids, submitted 